space. His "approximation theorem" and his "existence theorem" are the starting points of the modern study of moduli problems. Artin's contributions to the theory of surface singularities are of fundamental importance. In this theory he introduced several concepts that immediately became seminal to the field, such as the concepts of rational singularity and of fundamental cycles.

In yet another example of the sheer originality of his thinking, Artin broadened his reach to lay rigorous foundations to deformation theory. This is one of the main tools of classical algebraic geometry, which is the basis of the local theory of moduli of algebraic varieties.

Finally, his contribution to noncommutative algebra has been enormous. The entire subject changed after Artin's introduction of algebrogeometrical methods in this field. His characterization of Azumaya algebras in terms of polynomial identities, which is the content of the Artin-Procesi theorem, is one of the cornerstones in noncommutative algebra. The Artin-Stafford theorem, stating that every integral projective curve is commutative, is one of the most important achievements in noncommutative algebraic geometry.

Artin's mathematical accomplishments are astonishing for their depth and their scope. He is one of the great geometers of the twentieth century.

\section{Biographical Sketches}

George Mostow received his Ph.D. from Harvard University in 1948. He has served on the faculties of Johns Hopkins University (1952-1961) and Yale University (1961-1999). He is a member of the National Academy of Sciences (elected 1974) and has served as the president of the American Mathematical Society (1987-1988). He was awarded the 1993 Leroy P. Steele Prize for Seminal Contribution to Research for his book Strong Rigidity of Locally Symmetric Spaces (1973).

Michael Artin was born in Hamburg, Germany, in 1934, son of mathematician Emil Artin. He received his Ph.D. from Harvard University in 1960. He has been affiliated with the Institut des Hautes Études Scientifiques, as well as with MIT, whose faculty he joined in 1963. He was awarded the Steele Prize for Lifetime Achievement in 2002. In 2005 he received the Harvard Centennial Medal. He served as president of the AMS in 1991-1992. He is a member of the National Academy of Sciences and a fellow of the American Academy of Arts and Sciences, the American Association for the Advancement of Science, the Society for Industrial and Applied Mathematics, and the American Mathematical Society.

Israeli President Shimon Peres will present the prizes in May at a special session hosted by the Knesset.

\title{
Daubechies and Mumford Receive BBVA Foundation Award
}

INGRID DAUBECHIES of Duke University and DAVID MUMFORD of Brown University have been honored with the BBVA Foundation Frontiers of Knowledge Award in the Basic Sciences category for their work in pure mathematics, which, according to the prize citation, has "strongly influenced diverse fields of application, ranging from data compression to pattern recognition." Both researchers have formulated solutions to varied and complex problems starting from the vantage point of pure mathematics but guided by a multidisciplinary approach. The award carries a cash prize of 400,000 euros (approximately US\$550,000).

The jury singled out Daubechies, who trained as a physicist, for her work on "wavelets, which resulted in a new approach to data compression, with

DOI: http://dx.doi.org/10.1090/noti986 a strong impact on a multitude of technologies, including efficient audio and video transmission and medical imaging." Mumford's mathematical research has had considerable influence among theoretical physicists. He was recognized particularly for "his contributions to algebraic geometry and to the mathematics of computer vision. He has applied tools of variational calculus to the theory of vision and developed statistical models for imaging and pattern recognition. His work has had a lasting impact in both pure and applied mathematics."

A leader in the field of algebraic geometry, Mumford turned in the 1980s to a new problem: how to mathematically render the human ability to understand an image. One of his insights was that the brain operates by combining previous knowledge with what it is perceiving right now. His 

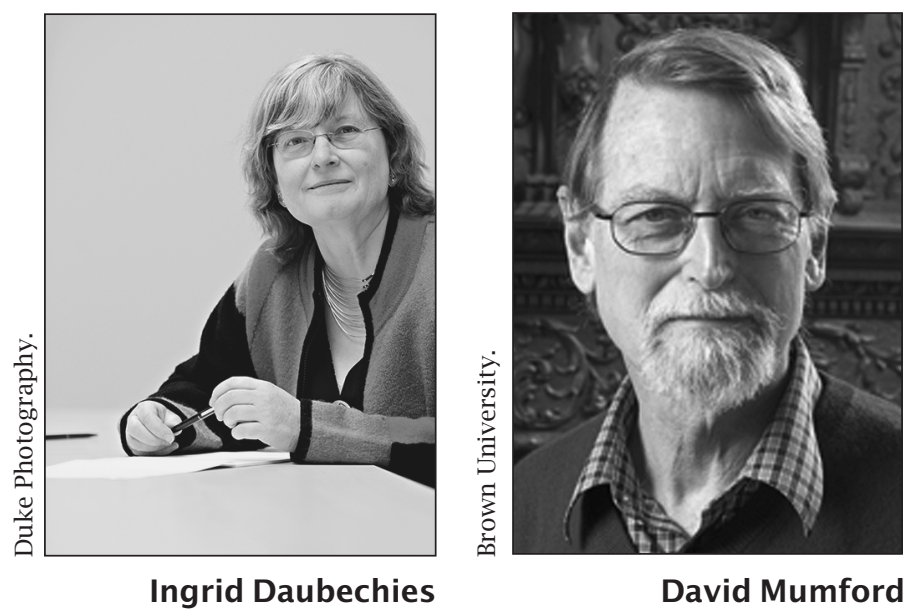

mission, now being advanced by his students, is to describe this human ability in mathematical terms.

Daubechies is a theoretical physicist who moved into mathematics because of theoretical physics' urgent need for new mathematical tools and models. Wavelets form a tool that allows the deconstruction of a mathematical object or an image, for example, into simpler components. In practical terms, this deconstruction means that information-rich images can be transmitted with no loss of quality. Daubechies's work on wavelets has one of its best-known applications in the JPEG 2000 image compression standard, but it is also a powerful instrument for testing theorems in basic research in pure mathematics.

Mumford and Daubechies have both served as president of the International Mathematical Union (IMU): Mumford from 1994 to 1998, and Daubechies from 2010 to the present.

Ingrid Daubechies was born in Houthalen, Belgium, in 1954. She received her Ph.D. in theoretical physics in 1980 from Vrije Universiteit Brussel and remained at that institution as a researcher until 1987. She then worked at AT\&T Bell Laboratories in New Jersey and joined the faculty of Princeton University in 1993. She has been professor of mathematics at Duke since 2011. She held a MacArthur Foundation Fellowship from 1992 to 1997. She was awarded the Steele Prize for Exposition in 1994, and in that same year gave a plenary lecture at the ICM in Zurich. She was awarded the Ruth Little Satter Prize in 1997. She received the National Academy of Sciences award in 2000, the first woman to be so honored. She was the AMS Gibbs Lecturer in 2005 and the AWM Noether Lecturer in 2006. She was elected to the American Academy of Arts and Sciences in 1993 and to the National Academy of Sciences in 1998.

David Mumford was born in Sussex, United Kingdom, in 1937. He received his Ph.D. from Harvard University in 1961. He taught at Harvard from 1961 to 1997, then joined Brown, from which he retired in 2007. He has held many visiting professorships at such institutions as the University of
Tokyo, the Tata Institute of Fundamental Research, the University of Warwick, and the Institut des Hautes Études Scientifiques. He held a MacArthur Foundation Fellowship from 1987 to 1992. He was awarded the Fields Medal in 1974, was cowinner of the 2006 Shaw Prize in Mathematical Sciences, and received the 2007 Steele Prize for Mathematical Exposition.

The BBVA Foundation also awarded a Frontiers of Knowledge Award in the Information and Communication Technologies to LOTFI A. ZADEH of the University of California Berkeley, an electrical engineer whose work involves the mathematical sciences, "for the invention and development of fuzzy logic." This "revolutionary" breakthrough, affirms the jury in its citation, has enabled machines to work with imprecise concepts in the same way humans do and to thus secure more efficient results more aligned with reality.

The BBVA Foundation engages in the promotion of research, advanced training, and the transmission of scientific knowledge to society at large, focusing especially on the analysis of emerging issues in five strategic areas: environment, biomedicine and health, economy and society, basic sciences and technology, and arts and humanities.

- Elaine Kehoe 